\title{
Technology of Biosurfactants for the Development of Environmental Remediation Processes
}

\author{
Verónica Leticia Colin* \\ Planta Piloto de Procesos Industriales y Microbiológicos (PROIMI), CONICET, Av. Belgrano y Pasaje Caseros, 4000, Tucumán, Argentina
}

The world is now confronted with serious problems of environmental contamination, which demand immediate solutions. The extensive production and use of hydrocarbons has resulted in widespread environmental contamination by these chemicals, which due to their toxicity on living organisms, are considered as proprietary pollutants. On the other hand, industrial and mining activities are important for economic development. However, these activities represent the main sources of heavy metal contamination, which provide unique challenges for their remediation, as they cannot be degraded into innocuous products. Although a variety of remediation technologies that include, mostly physicochemical methods, are available to address contamination with hydrocarbon and heavy metal, these processes have several disadvantages including the high cost and the risk of secondary environmental pollution. The situation is more critical in developing countries where there is no legislation to respect. As a result, it remains important to develop new techniques for reduction of these pollutants to acceptable levels, but at more manageable costs.

The microbial remediation, known as bioremediation, offers high specificity in the removal of particular pollutants while also offering operational flexibility. Scientific evidence indicates that the microbial activities can be exploited in the bioremediation of organic- and metalcontaminated sites. Thus, the comprehensive overview of advances in this field provides a very encouraging picture. For example, technologies involving microorganisms are successfully applied to waste streams such as sewage sludge, industrial effluents, and mine water. However, the use of microbial products instead of whole-cell microbes for environmental remediation could have unquestionable advantages, since microorganisms do not need to have the ability to survive in the contaminated sites. Microbial products as some enzymes and/or certain exopolymers as the biosurfactants, are among the constituents of the first cellular defence line against diverse toxics. Biosurfactants are a structurally diverse group of surface-active substances produced by microorganisms, which facilitate the uptake of water-insoluble substrates. They can be used therefore, to release hydrocarbon pollutants characterized by low solubility, from soil and other media. There are also reports in the literature regarding the removal of heavy metals from wastewater and soils using biological agents as biosurfactants. They can increase desorption of the soilbound metals and facilitate their transport through the soil matrix, in a process called washing. Compared to their synthetically produced counterparts whom it is characterized by a high production costs that make them unaffordable for use in larger ecosystems, surfactants of microbial origin are more economically viable. In addition, their greater biodegradability and their reduced toxicity are in agreement with the global trend of replacing synthetic compounds by those derivates of natural source. However, some reports indicate that under certain circumstances biosurfactants can be toxic to the environment.

In view of the wide range of technological applications of the biosurfactants, researchers have emphasized the key parameters affecting their production in terms of higher yields and lower production costs. Many studies suggest that the inclusion of hydrocarbons in the growth medium markedly increases the synthesis of biosurfactants, but this also creates a challenge in terms of subsequent separation of the compound produced. Therefore, the main strategy to achieve this are through assessment of the appropriate organism, and the use of cheap or waste substrates to lower the initial raw material costs involved in the process, including optimization of the culture conditions and cost-effective separation processes to maximize recovery of the final product. Another important aspect regarding biological remediation technologies is the use of biosurfactant in the process on a large scale. Little is also known about the potential of biosurfactant production by microorganisms in situ, since the most of the described studies were done under laboratory conditions.

In summary, there are many unexplored edges on this topic. It is expected that the concerted efforts of the lines of research involved provide valuable progress to the short term. However, progressive entry of biosurfactants derived mainly from renewable raw materials into the market is, at present, a concrete fact.

*Corresponding author: Verónica Leticia Colin, Planta Piloto de Procesos Industriales y Microbiológicos (PROIMI), CONICET, Av. Belgrano y Pasaje Caseros, 4000, Tucumán, Argentina, Tel: + 54-381-4344888, ext. 26; Fax: + 54381-4344887; E-mail: veronicacollin@yahoo.com.ar

Received April 20, 2012; Accepted April 23, 2012; Published April 23, 2012

Citation: Colin VL (2012) Technology of Biosurfactants for the Development of Environmental Remediation Processes. Single Cell Biolo 1:e107. doi:10.4172/21689431.1000e107

Copyright: (c) 2012 Colin VL. This is an open-access article distributed under the terms of the Creative Commons Attribution License, which permits unrestricted use, distribution, and reproduction in any medium, provided the original author and source are credited. 